\title{
Spatial aliasing effects on beamforming performance in large-spacing antenna array
}

\author{
Dwi Joko Suroso a,, , Deepak Gautam ${ }^{\mathrm{b}}$, Sunarno ${ }^{\mathrm{a}}$ \\ ${ }^{a}$ Department of Nuclear and Engineering Physics, Universitas Gadjah Mada,, Yogyakarta, 55281,Indonesia \\ ${ }^{b}$ Department of Transdisciplinary Science and Engineering, Tokyo Institute of Technology, Tokyo, 152-8552, Japan
}

\section{Article history:}

Received: 15 April 2019 / Received in revised form: 13 May 2019 / Accepted: 20 May 2019

\begin{abstract}
In the next wireless communication generation, 5G, the half-lambda inter-element spacing as high-resolution antenna array is usually employed due to its benefits i.e., minimum grating lobes and providing one end-fire maximum. However, to compensate the lower aperture from a such short-spacing elements, the number of antennas should be grown larger. It will be costly and increase the complexity in terms of antenna array analysis. In this paper, the aliasing effects on beamforming of antenna array geometry utilizing inter-element spacing more than half-lambda is presented. The geometry of linear, circular and planar antenna arrays will be explored and simulated at center frequency of $60 \mathrm{GHz}$. It is also due the fact that many researchers working on $5 \mathrm{G}$ believe that $60 \mathrm{GHz}$ will be employed as $5 \mathrm{G}$ frequency band. The frequency of $60 \mathrm{GHz}$ is truly higher than today long-term-evolution (LTE) working frequency and it is really challenging to model its signal due to small wavelength and its effective signal working distance as effect of rain attenuation, etc. As our preliminary results, linear array, which only considers the azimuthal, the spatial aliasing appears in the inter-element distance more than 1-lambda. On the other hand, the circular and planar antenna array consider the azimuth and elevation properties of incoming signals. In circular array, the angular power of a signal can be detected accurately applying the 3-sector antenna pattern. When the inter-element distance grows more than 1.5 lambda, the spatial aliasing which appear as side lobes with similar angular power dominate the incoming signal detection. The result shows us that employing the 2-lambda distance or more will be useless. Planar array which actually a 2-axis linear array give the unexpected results, most of detections are inaccurate and angular power also low. This concludes that spatial aliasing effects will degrade the beamforming performance due to confusion between real signal and fake signal resulting from similar values of array factor.
\end{abstract}

Keywords: 5G, antenna array, spatial aliasing, beamforming.

\section{Introduction}

Some researchers published their results on 28 and $60 \mathrm{GHz}$ frequency band, or millimeter wave, to be suitable for 5G [13]. The frequency spectrum of $60 \mathrm{GHz}$ has large freebandwidth which can support $5 \mathrm{G}$ data rate speed requirement. Rain attenuation and working distance effects on communications signal in $60 \mathrm{GHz}$ are proven to be negligible when applied working distance less than $200 \mathrm{~m}$ [4-6]. However, utilizing this high frequency also gives challenges, especially in the antenna design and in its signal modelling [7-8]. Therefore, beamforming performance needs to be taken into account for this really small wavelength signal which correspond to the size of antenna array [9].

For more than two decades, multiple-input multiple-output (MIMO) system utilizing antenna arrays have been proven to show the better link performance and channel capacity even with the limited bandwidth [10-12]. In 5G, the Massive MIMO system utilizing massive antenna array is expected as one of promising technique in 5G. Massive MIMO system employs massive number of elements on antenna array both in * Corresponding author.

Email: dwi.jokosuroso@ugm.ac.id transmitter (TX) and receiver (RX) [13].

The mainstream researches in MIMO system use the halflambda antenna spacing or so-called the high-resolution antenna because of its directivity performance. But, as the spacing distance is short, the antenna aperture will be low. So, there is no question, there will be needed tens to hundreds of antenna elements [14-16].

In this paper, aside of mainstream line, we are looking forward to how the large spacing will be beneficial in $5 \mathrm{G}$, in terms of providing the large aperture antenna in MIMO system [17]. When array grows large, the interference effect between elements is negligible and its analysis becomes simple. Furthermore, many proofs show that by increasing the spacing elements of antenna array, the beamforming performance also improved. The beamforming is useful because the base station and mobile station/user should be driven or "steered" effectively in its beam directions. This will improve the quality of communication link which leads to higher capacity and data rates speed. The data rate speed up to $10 \mathrm{~Gb} / \mathrm{s} / \mathrm{Hz}$ is one of requirements in 5G [18-19].

By analyzing the spatial aliasing effects on beamforming, the effect of antenna geometries and their inter-element spacing can be observed. Spatial aliasing will be shown as the grating 
lobes which have similar properties to main lobe/beam when the inter-element spacing become larger. Since the array geometry will affect the array factor in terms of arrangement of antenna elements and radius or inter-element spacing distance. Thus, the certain antenna geometry and its inter-element distance for certain application employing beamforming technique can be selected.

\section{Materials and Methods}

\subsection{MIMO System and Antenna Array Geometry}

In introduction section, the MIMO system is briefly stated. This system is useful in terms of communication diversity and reliability of a channel. Transmitter (TX) and receiver (RX) utilize the multi-antenna system or antenna array by which the signal can be transmitted to each individual element in the same frequency band. In the receiver side, the incoming signals will be in the different power and travelling time. This is called the multipath propagation. The illustration of MIMO wireless communication can be shown in Fig. 1

Fig. 1. Illustration of MIMO wireless communication

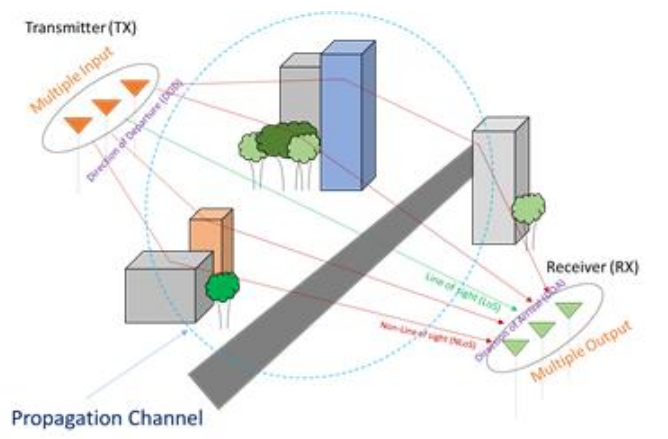

When beamforming algorithm is employed to MIMO system, the direction of signal travelling and receiving can be estimated. This beamforming algorithm will mainly depend on the antenna array factors. Antenna array factor itself will be affected by the antenna geometry and inter-element distance of array elements.

There will be three antenna array geometries to be discussed in this paper: linear, circular and planar arrays. Linear array is said as the simplest array geometry. The circular array, due to its round form, it can cover both azimuthal and co-elevation plane. Planar array comes in form of a plane or a rectangle. Circular and planar antenna are examples of 2-D antenna geometry.

\subsubsection{Linear Array}

The uniform spacing and antenna elements are assumed. It means that the antenna element is the same, in terms of its shape, polarization, and gain. This assumption will be also applied for circular and planar arrays in the following discussion.

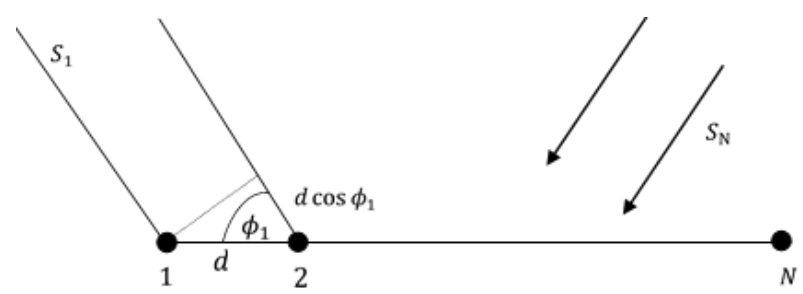

Fig. 2. Illustration of uniform linear array (ULA)

Array factor (AF) is a factor in the array antenna which is related to the number of elements and phases difference between elements produced by the inter-element distance. If the inter-element distance is uniform, denote by $d$, and the number of antenna elements is $N$, the expression of the AF of linear array in receiver side which laid in $x$ axis is [20]

$$
\boldsymbol{a}_{\mathrm{R}}(\phi)=\left[\begin{array}{c}
1 \\
\exp (-j k d \cos (\phi)) \\
\exp (-j 2 k d \cos (\phi)) \\
\vdots \\
\exp (-j(N-1) k d \cos (\phi))
\end{array}\right]
$$

\subsubsection{Circular Array}

Circular array has a round geometric shape. Circular array has ability to obtain 3D analysis for the travelling and incoming, Direction-of-Departure (DoD) or Direction-ofArrival (DoA), respectively [21].

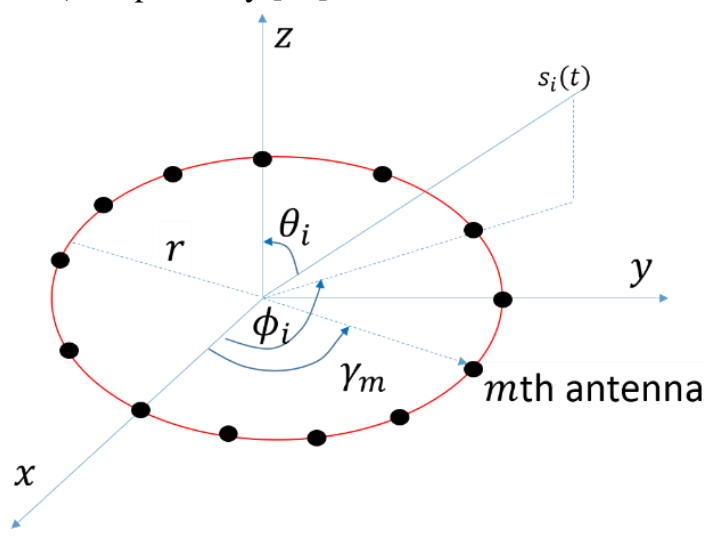

Fig. 3. Illustration of uniform circular array (UCA)

Where $r$ and $M$ are radius and total number of elements, respectively. The elements are assumed to be identical and omnidirectional. The elevation and azimuth angles of impinging signal denoted by $\theta \in[0, \pi / 2]$ and $\phi \in[0,2 \pi]$. The angle between the $m$ th element of array and the $x$-axis, for $m \in[0, M-1]$ is $\gamma_{m}=2 \pi(\mathrm{m} / M), m=1,2, \ldots \ldots, M, \quad m=$ $1,2, \ldots \ldots, M$. The phase difference (of the complex envelope of the field at $m$ th element with respect to the origin) is $\Delta \phi_{m, i}=$ $k r \sin \theta_{i} \cos \left(\phi_{i}-\gamma_{m}\right) . k=2 \pi / \lambda$, is the wave number, the 
element-space circular array factor in the receiver side or steering vector is

$$
\mathbf{a}\left(\theta_{i}, \phi_{i}\right)=\exp \left(j \Delta \phi_{m, i}\right)=\left[\begin{array}{c}
\exp \left(j k r \sin \theta_{i} \cos \left(\phi_{i}-\gamma_{0}\right)\right) \\
\exp \left(j k r \sin \theta_{i} \cos \left(\phi_{i}-\gamma_{1}\right)\right) \\
: \\
\cdot \\
\exp \left(j k r \sin \theta_{i} \cos \left(\phi_{i}-\gamma_{M-1}\right)\right)
\end{array}\right]
$$

\subsubsection{Planar Array}

Illustration of 2-D planar array is depicted in Figure 4. As mentioned before, the elements are uniform, they are arranged along a rectangular grid in the $x y$-plane, with an inter-element spacing $d_{x}$ in the $x$-direction and an inter-element spacing $d_{y}$ in the $y$-direction. To know the AF expression, the grid of $x-y$ plane can be used for assistance. This grid represents $m$ th element of $x$ axis and nth element of $y$-axis . Position vector can be described as

$$
\vec{r}_{m n}^{\prime}=x_{m n}^{\prime} \hat{x}+y_{m n}^{\prime} \hat{y}+z_{m n}^{\prime} \hat{z}
$$

Illustration of uniform planar array (UPA) can be depicted in Fig. 4.

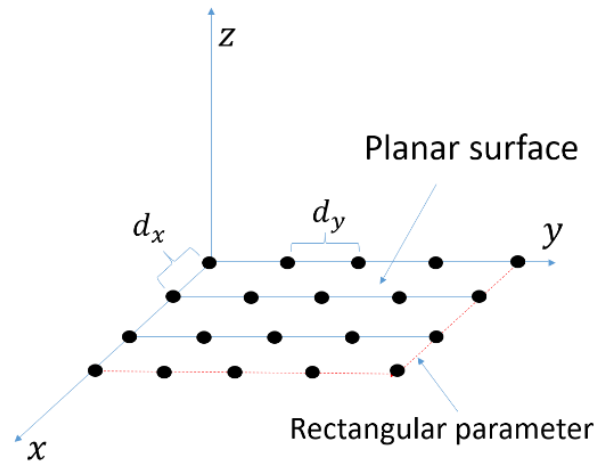

Fig. 4. Illustration of UPA

If we have already indicated the spacing, and the array starting from the origin, and only 2-D of $x$ and $y$ axes are considered, the position vector can be rewritten as

$$
r_{m n}^{\prime}=m d_{x} \hat{x}+n d_{y} \hat{y}
$$

where $m, n=1,2,3, \ldots$. Based on the sphere coordinate of position vector, we can have AF by splitting along with the two dimensions as follows

$$
a(\theta, \phi)=\sum_{n=0}^{N-1} \sum_{m=0}^{M-1} I_{m n} e^{j k\left(m d_{x} \sin \theta \cos \phi+n d_{y} \sin \theta \sin \phi\right)}
$$

Here, $I_{m n}$ refers to the excitation of signal amplitude, or energy applied to certain amplitude, of the $m n$th element of the array, and is assumed as a real number yielding broadside radiation. Separating the array factor into dimension can be done if the condition of excitation follows $I_{m n}=I_{m x} I_{y n}$. This means that the excitation is product of two direction, $x$ and $y$ direction. In each direction is common to use uniform amplitude but progressive phase in each direction which can be written as $I_{m x}=I_{0} e^{j m \alpha_{x}}$ and $I_{y n}=I_{0} e^{j n \alpha_{y}}$ where $\alpha_{x}$ and $\alpha_{y}$ are the phase gradients in the respective directions. From here, the AF then can be calculated by

$a(\theta, \phi)=I_{0} \sum_{n=0}^{N-1} e^{j k\left(m d_{x} \sin \theta \cos \phi+\alpha_{x}\right)} \sum_{m=0}^{M-1} e^{j k\left(n d_{y} \sin \theta \sin \phi+\alpha_{y}\right)}$

or we can simply call this as the product of two array factors; array factor in linear $x$ direction and linear $y$ direction. This also can be used in different pair of axes based on the position vector in sphere coordinate [22].

\subsection{Beamforming}

Antenna array or multi antenna system is the basic idea behind MIMO system establishment. By employing antenna array, the data speed rate increases, thanks to the multiplexing and diversity gain MIMO system offered. Direction-of-Arrival (DoA) estimation algorithm is still alive topic in array signal processing up to now. Beamforming method, which is also related to DoA, is a method to "steer" the traveling signal and/or received signal, then calculate its power. When the "steered" coincides with the DoA of signals the maximum power will be obtained. The main purpose of beamforming is to increase the signal transmission efficiency, especially in short-wavelength communication i.e. in $60 \mathrm{GHz}$ which need directive signal.

In beamforming, the complex weight has a significant role as the signal will be multiplied by this weight. It will adjust the magnitude and phase of the signal from each antenna. Thus, the output of array antenna can be formed to transmit or receive the desired beam in specific direction and minimize the other directions.

For the beamforming technique, the model of single output signal can be expressed as

$$
\boldsymbol{y}(t)=\mathbf{w}^{\boldsymbol{H}} \mathbf{x}(\mathrm{t})
$$

From (7) we can basically design the weight, $\boldsymbol{w}$. The total averaged output power over L realizations for an array antenna can be written as

$$
\begin{aligned}
\boldsymbol{P}(\boldsymbol{w}) & =\frac{1}{L} \sum_{l=1}^{L}\left|y\left(t_{l}\right)\right|^{2} \\
& =\frac{1}{L} \sum_{l=1}^{L} \mathbf{w}^{\boldsymbol{H}} \mathbf{x}\left(\mathbf{t}_{l}\right) \mathbf{x}^{\boldsymbol{H}}\left(\mathbf{t}_{l}\right) \mathbf{w} \\
& =\mathbf{w}^{\boldsymbol{H}} \widetilde{\boldsymbol{R}}_{\boldsymbol{x} x} \mathbf{w}
\end{aligned}
$$

where $\widetilde{\boldsymbol{R}}_{\boldsymbol{x} \boldsymbol{x}}$ is correlation matrix. This correlation matrix is useful to distinguish real signal angle and noise of the signal. In MIMO system, the correlation matrix can be defined as

$$
R_{x x}=E\left[\mathbf{x}(\mathrm{t}) \mathbf{x}^{H}(t)\right]
$$


Signal component from transmitter, $\boldsymbol{X}$, is the transpose of all signal from $M$ number of elements.

$$
\begin{gathered}
X=\left[x\left(t_{1}\right) x\left(t_{2}\right) x\left(t_{3}\right) \ldots \ldots x\left(t_{M}\right)\right]^{T} \\
X=A \cdot\left[\begin{array}{c}
\left.s\left(t_{1}\right) s\left(t_{2}\right) s\left(t_{3}\right) \ldots \ldots s\left(t_{M}\right)\right]^{T} \\
+\left[n\left(t_{1}\right) n\left(t_{2}\right) n\left(t_{3}\right) \ldots \ldots n\left(t_{M}\right)\right]^{T}
\end{array}\right. \\
X=A \cdot S+N
\end{gathered}
$$

Here $n$ is a noise-corrupted signal, in simulation it can be done by number of realizations $(L=10,000$ realization is commonly used as this give the randomized signal to be noisecorrupted-like signal).

Then,

$$
\boldsymbol{R}_{\boldsymbol{x} \boldsymbol{x}} \approx \tilde{R}_{x x}=\frac{1}{L} \sum_{l=1}^{L} \mathbf{x}\left(\mathrm{t}_{l}\right) \mathbf{x}^{H}\left(t_{l}\right)=\frac{1}{L} \boldsymbol{X} \boldsymbol{X}^{H}
$$

The

weight

on beamforming can be assumed as the array steering vector in certain scanning direction. For example, for uniform linear array (ULA) the scan angle will be only the azimuthal dimension. For the 2D array, it will be both azimuthal and coelevation, etc. In this explanation we will take the simple one scan direction in $\theta$ direction.

$$
\boldsymbol{w}=\boldsymbol{a}(\boldsymbol{\theta})
$$

As aforementioned, if the angle of incident signal is the same as the DoA signal, the output power will be maximum, $\theta=$ $\theta_{i} \rightarrow P(\theta)$ maximum. It means that, $w=a\left(\theta-\theta_{i}\right)$ aligned the phase of the signal components received by all elements, then add constructively to produce a maximum power. Weight can be normalized as

$$
\boldsymbol{w}=\boldsymbol{a}(\boldsymbol{\theta})=\frac{\boldsymbol{a}(\boldsymbol{\theta})}{\sqrt{\boldsymbol{a}^{H}(\boldsymbol{\theta}) \boldsymbol{a}(\boldsymbol{\theta})}}
$$

Substitute (14) to (8) will result the output power as a function of angle of arrival,

$$
P(\theta)=\frac{\boldsymbol{a}^{H}(\boldsymbol{\theta}) \tilde{R}_{x x} \boldsymbol{a}(\boldsymbol{\theta})}{\boldsymbol{a}^{H}(\boldsymbol{\theta}) \boldsymbol{a}(\boldsymbol{\theta})}
$$

Capon's Beamformer or largely known as Minimum Variance Method (MVM) is the modified of conventional beamforming which is

$$
P_{M V M}(\theta)=\frac{1}{\boldsymbol{a}^{H}(\boldsymbol{\theta}) \tilde{R}_{x x}{ }^{-1} \boldsymbol{a}(\boldsymbol{\theta})}
$$

Thus, in beamforming, the weight can be interpreted as the spatial filter, matched the impinging signal, and attenuate the output power which not coming from the angles of the incoming signal [23].

\subsection{Simulation Environment}

\subsubsection{Antenna Pattern for UCA}

The assumption of antenna pattern use for UCA is based on the 3GPP antenna pattern (azimuth only) which is the 3-sector UCA is utilized. If there is no pattern applied, the beamforming result is unrealistic. This antenna pattern is specified by

$$
G(\varphi)=-\min \left[12\left(\frac{\varphi}{\varphi_{3 d B}}\right)^{2}, G_{m}\right] \text { where }-180 \leq \varphi \leq 180
$$

$\varphi_{3 d B}$ is the $3 \mathrm{~dB}$ beamwidth, $G_{m}$ is the maximum attenuation. For this 3-sector antenna, $\varphi_{3 d B}=70 \mathrm{~dB}$ and $G_{m}=$ $20 \mathrm{~dB}[24]$.

\subsubsection{Noise Assumption}

The incident signal assumed has random phase (incoherent), the steering vector of this incident signal based on the array geometry. Noise is randomized based on the noise covariance, $\sigma^{2}$, and assumed to be $20 \mathrm{~dB}$. To make realizations, the phase of the incident signal and the noise is randomized to $L=$ 10,000 realizations and number of elements in array, $N$ [23].

\subsubsection{Simulation Parameters}

Some simulation parameters are given in Table 1. Some of the parameters, basically follow the trend of the next wireless communication trend e.g., frequency bands and inter-element spacing distance. Frequency $60 \mathrm{GHz}$ is selected here to only know how the lambda can affect to the size of array. Basically, the frequency can be chosen any number, because in this simulation, the constrain is only the inter-element distance not the power of received signal related to working frequency.

The inter-element distance of 0.5, 1, 1.5 and 2-lambda are simulated to know the effect of spatial aliasing resulted of this distance to the beamforming performance. The elements of antenna are uniform, omnidirectional to make analysis simple. As the TX and RX are assumed to have a great distance and no scattering effects near RX. The plane wave assumption is also applied.

Table 1. Simulation parameters

\begin{tabular}{lll}
\hline No & Parameter & \multicolumn{1}{c}{ Value } \\
\hline 1. & Frequency & $60 \mathrm{GHz}$ \\
2. & No. of elements & 10 \\
3. & Inter-element distance & $(0.5,1,1.5,2)$ lambda \\
4. & Antenna Type & Uniform, omnidirectional \\
5. & Wave Assumption & Plane wave \\
6. & Beamforming method & Conventional and MVM
\end{tabular}




\section{Results and Discussion}

In this result and discussion, the geometry and inter element distance of an array are emphasized.

\subsection{ULA Beamforming}

The 10-element ULA is simulated and the beamforming performance is compared between $0.5,1,1.5$ and 2-lambda. The known incoming signals for simulation are $10^{\circ}, 45^{\circ}$ and $72^{\circ}$. Take a note that in this ULA simulation, the antenna pattern is assumed to be omnidirectional. The antenna pattern mentioned in the previous section is for UCA only. It is used for validating the steering vector of UCA using an antenna pattern.

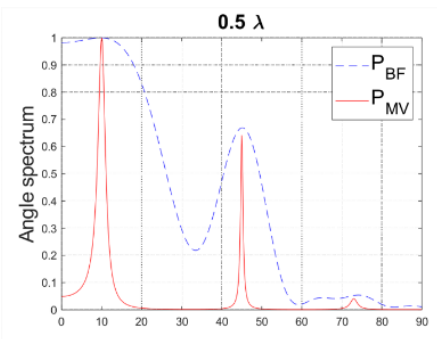

$1.5 \lambda$

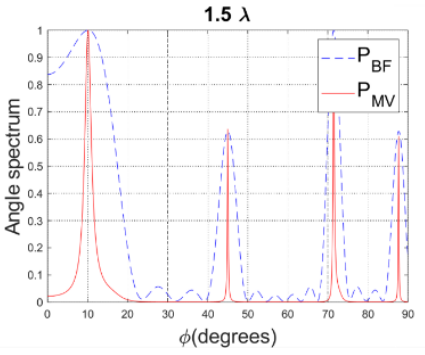

Fig. 5. Results of ULA beamforming

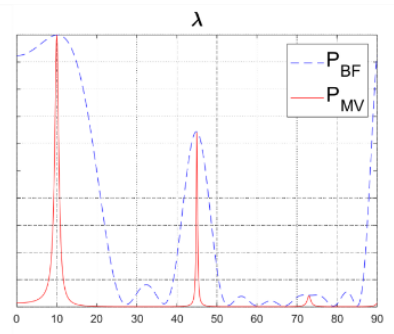

$2 \lambda$

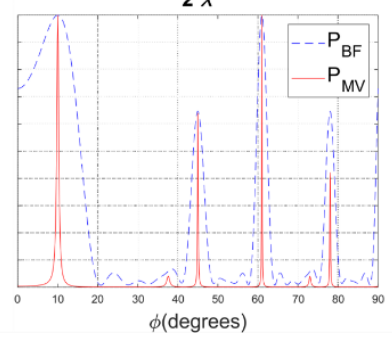

Fig. 5 shows that the inter-element distance has impact in the accuracy of beamforming, seen as the detection of incoming signal become sharper. However, by increasing this element distance the side beams appear to be a distraction since the size and direction is similar to main beam. This is the spatialaliasing that will degrade the performance of DoA estimation and the beamforming results. ULA result in Fig. 5 shows the normalization results of angular power spectrum. This purposes for easier reading of the peak of the beamforming in regards of incoming signals.

\subsection{UCA Beamforming}

In UCA, the 3-sector antenna pattern is used for antenna pattern assumption for each individual element. The results are quite realistic since the element spacing larger than half-lambda giving sharper angular power spectrum to the specified incoming signal $\left(\theta=45^{\circ}\right.$ dan $\left.\varphi=100^{\circ}\right)$. Furthermore, the spacing more than 1.5 half lambda may not be useful. Fig. 6 depicts the result for UCA with four different inter-element spacing distance. The color bar is showing the angular power spectrum in dB. As we can see from Fig.6 that the DoA estimation of incoming signal can be done accurately. The improvement of DoA estimation related to beamforming can be observed when the inter-element distance equal to lambda, the peak of detected signal is sharper than in half-lambda. The spatial aliasing power is also still low compared to the angular power of detected signal. For the 1.5-2 lambda, the spatial aliasing occurs and has power similar to detected signal. This effect on inter-element distance more than lambda degrades the beamforming performance [25].
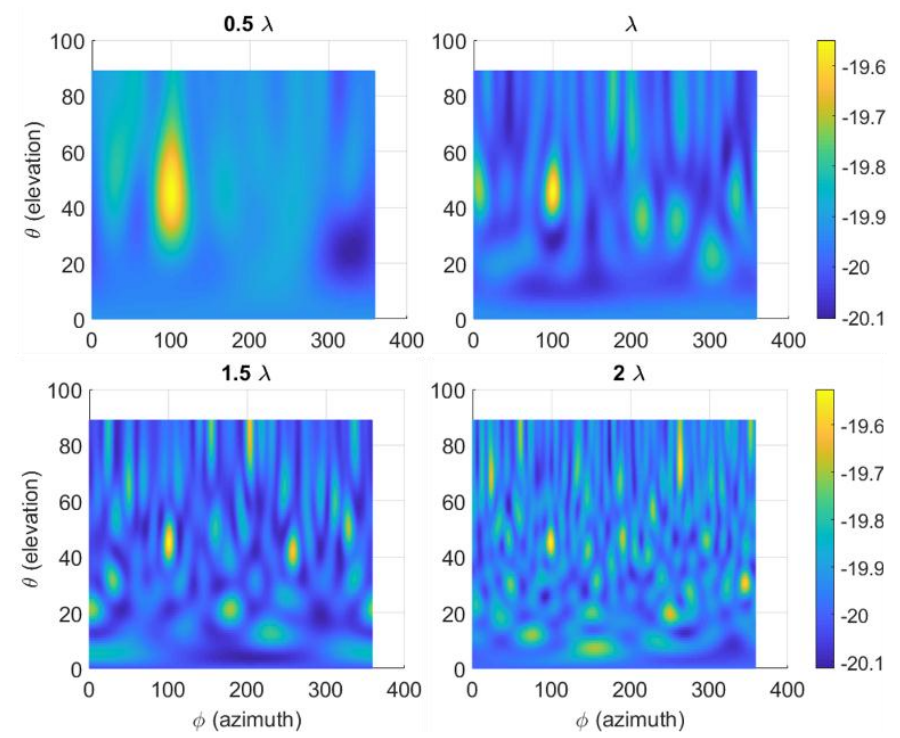

Fig. 6. Results of UCA Beamforming
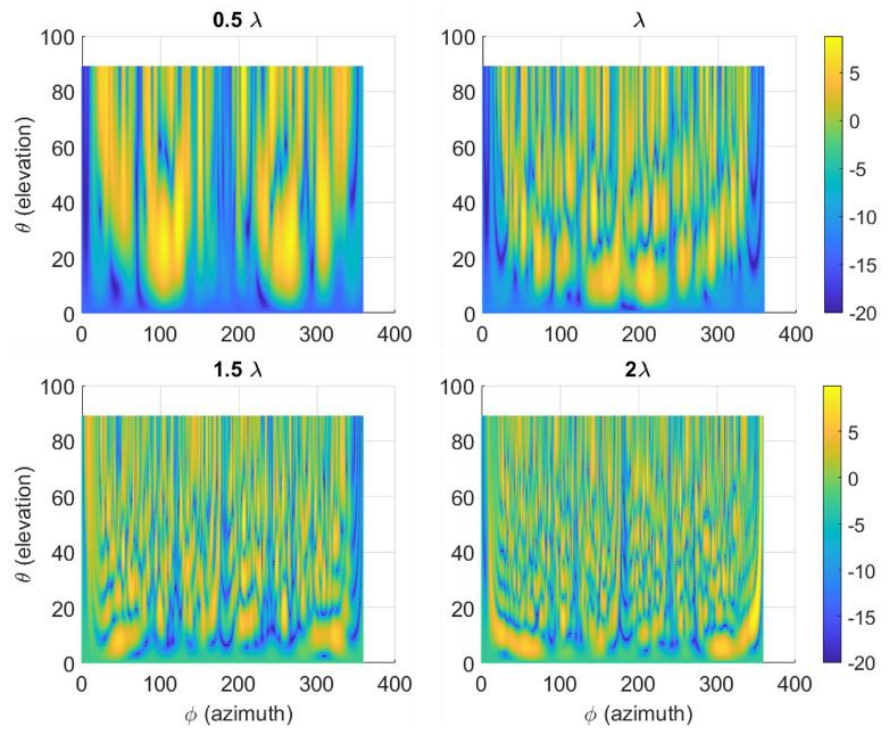

Fig. 7. Results of UPA beamforming

\subsection{UPA Beamforming}

In UPA, the omnidirectional antenna pattern is assumed. The UPA is traditionally similar to ULA placing in two-axes direction. This gives challenge in the terms of antenna factor or steering vector. The assumed received signal has same angle with those in UCA; $\theta=45^{\circ}$ dan $\varphi=100^{\circ}$. Fig. 7 shows the results of UPA. The results show that in the same incoming signal of UCA, the antenna geometry cannot perform well in 
all inter-element spacing distance variation. There are lowaccuracies, even in the half-lambda spacing, and the distance more than half-lambda is unusable. From Fig. 7, signal cannot be detected because of spatial aliasing in almost all directions.

\section{Conclusion and Future Works}

The spatial aliasing can affect the beamforming performance in the large-spacing antenna array. This spatial aliasing can be seen from the peak of side lobe which distract the directional estimation to similar power of the main lobe in beamforming. The geometry of antenna array which the interelement spacing distance is varied gives several insights on how the distance and arrangement can really affect how antenna will work, especially in directing the signals. Beamforming results of ULA, UCA and UPA are presented based on only simulation on the antenna theory. The ULA and UCA results show that the inter-element distance equals to lambda improving the beamforming, more than that the spatial aliasing effects formed. UPA in our simulation gives the unexpected results since the inter-element distance variation does not obtain accurate signal detection. From the discussion, the small-lambda signal i.e., in $60 \mathrm{GHz}$ becomes the challenge for the antenna designer to take into account the issues of antenna geometry and inter-element distance. For future work, the comparison of beamforming algorithm between simulation and field experiment will be conducted.

\section{Acknowledgment}

Our great appreciation goes to Dr. Azril Haniz from National Institute of Information and Communications Technology (NICT), Japan and Dr. Panawit Hanpinitsak from Tokyo Institute of Technology, Japan for providing very valuable discussion related to Beamforming algorithm used in this paper.

\section{References}

1. T. S. Rappaport, et al., Millimeter Wave mobile communications for $5 G$ cellular: It Will Work!, IEEE Access 1 (2013) 335-349.

2. T. S. Rappaport, J. N. Murdock, and F. Gutierrez, State of the art in 60GHz integrated circuits and systems for wireless communications, Proc. IEEE, 2011, pp. 1390-1436.

3. S. Seidel and H. Arnold, Propagation measurements at $28 \mathrm{GHz}$ to investigate the performance of local multipoint distribution service (LMDS), Proc. GLOBE- COM, 1995, pp. 754-757.

4. F. Molisch and F. Tufvesson, Propagation Channel Models for NextGeneration Wireless Communications Systems, IEICE Trans. Commun., 97 (2014) 2022-2034.

5. K. Wangchuk, K. Umeki, T. Iwata, P. Hanpinitsak, M. Kim, K. Saito, and J. Takada, Double directional millimeter wave propagation channel measure- ment and polarimetric cluster properties in outdoor urban picocell environment, IEICE Trans. Commun. (2017).
6. A. I. Sulyman, A. Alwarafy, G. R. MacCartney, T. S. Rappaport, and A. Alsanie, Directional radio propagation path loss models for millimeterwave wireless networks in the 28-, 60-, and 73-ghz bands, IEEE Trans. Wirel. Commun. 15 (2016) 6939-6947.

7. A. Gupta and R. K. Jha. A survey of $5 \mathrm{~g}$ network: architecture and emerging technologies, IEEE Access 3 (2015) 1206 - 1232.

8. F. Rusek, et.al, Scaling up MIMO: opportunities and challenges with very large arrays, IEEE Signal Process. Mag. 30 (2013) 4060.

9. S. Kutty and D. Sen, Beamforming for millimeter wave communications: An inclusive survey, IEEE Commun. Surveys Tut. 18 (2016) 949-973.

10. G. J. Foschini and M. J. Gans, On limits of wireless communications in a fading environment when using multiple antennas, Wireless Personal Commun. 6 (1998) 311-335.

11. I. E. Telatar, Capacity of multi-antenna Gaussian channels, European Trans. Tel. 10 (1999) 585-595.

12. D. S. Shui, G. J. Foschini, M. J. Gans, and J. M. Kahn, Fading correlation and its effect on the capacity of multielement antenna systems, IEEE Trans. on Commun. 48 (2000) 502-513.

13. X. Gao, et.al., Massive MIMO in Real Propagation Environments: Do All Antennas Contribute Equally?, IEEE (2015) 3917-3928.

14. J. Hoydis, S. Ten Brink, and M. Debbah, Massive MIMO: How many antennas do we need?, Communication, Control, and Computing (Allerton), 49th Annual Allerton Conference on. IEEE, 2011, pp. 545-550.

15. H. Huh, G. Caire, H. C. Papadopoulos, and S. A. Ramprashad, Achieving massive MIMO spectral efficiency with a not-so-large number of antennas. IEEE Transactions on Wireless Communications 11 (2012) 3226-3239.

16. Y. H. Nam, B. L. Ng, K. Sayana, Y. Li, J. Zhang, Y. Kim, and J. Lee, Fulldimension MIMO (FD-MIMO) for next generation cellular technology, IEEE Communications Magazine 51 (2013) 172-179.

17. D.J. Suroso, K. Saito and J. Takada, On ilnvestigation of directional channel model for large spacing array MIMO: eigenvalues comparison of measurement and channel synthesis, IEICE Technical Report (2018) 1-6.

18. Y. Konishi, et. al., Versatile radio channel sounder for double directional and multi-link MIMO channel measurements at $11 \mathrm{GHz}$, IEICE TRANSACTIONS on Electronics 97 (2014) 994-1004.

19. M. Kim, J. Takada, Y. Chang, J. Shen and Y. Oda, Large scale characteristics of urban cellular wideband channels at $11 \mathrm{GHz}$, 9th European Conference on Antennas and Propagation (EuCAP), Lisbon, 2015, pp. 1-4.

20. Andreas Molisch, Wireless Communication, IEEE, 2nd Edition, 2010

21. S.O. Ata and C. Isik, High-Resolution Direction-of-Arrival Estimation via Concentric Circular Arrays, ISRN Signal Processing, Hindawi Publishing Corporation, 2013.

22. S. V. Hum and J. Perruisseau-Carrier, Reconfigurable reflectarrays and array lenses for dynamic antenna beam control: A review, IEEE Transactions on Antennas and Propagation 62 (2014) 183-198.

23. Z. Chen, et.al., Introduction to Direction-of-Arrival Estimation, Artech House, 2010.

24. 3rd Generation Partnership Project; Technical Specification Group Radio Access Network, Spatial channel model for Multiple Input Multiple Output (MIMO) simulations (Release 9), 3GPP Technical Report, 2009.

25. N.H.M. Adnan, et.al., Effects of inter element spacing on large antenna array characteristics, Proc. of the 4th IEEE International Conference on Smart Instrumentation, Measurement and Applications (ICSIMA), 2017. 